\title{
Electrical characterization of single nanometer-wide Si fins in dense arrays
}

\author{
Steven Folkersma ${ }^{* 1,2}$, Janusz Bogdanowicz ${ }^{1}$, Andreas Schulze ${ }^{1}$, Paola Favia ${ }^{1}$, \\ Dirch H. Petersen ${ }^{3}$, Ole Hansen ${ }^{3}$, Henrik H. Henrichsen ${ }^{4}$, Peter F. Nielsen ${ }^{4}$, Lior Shiv ${ }^{4}$ \\ and Wilfried Vandervorst ${ }^{1,2}$
}

\section{Full Research Paper}

\section{Address:}

${ }^{1}$ IMEC, Kapeldreef 75, B-3000 Leuven, Belgium, ${ }^{2}$ Instituut voor Kernen Stralingsfysika, KU Leuven, Celestijnenlaan 200D, B-3001 Leuven, Belgium, ${ }^{3}$ Department of Micro- and Nanotechnology, Technical University of Denmark, DTU Nanotech Building 345 East, DK-2800 Kgs. Lyngby, Denmark and ${ }^{4}$ CAPRES A/S, Scion-DTU, Building 373 , DK-2800 Kgs. Lyngby, Denmark

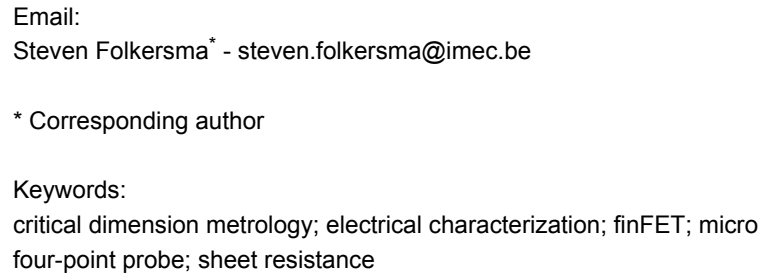

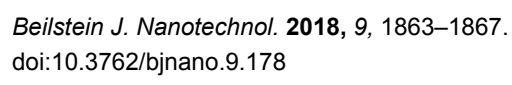

\begin{abstract}
This paper demonstrates the development of a methodology using the micro four-point probe $(\mu 4 \mathrm{PP})$ technique to electrically characterize single nanometer-wide fins arranged in dense arrays. We show that through the concept of carefully controlling the electrical contact formation process, the electrical measurement can be confined to one individual fin although the used measurement electrodes physically contact more than one fin. We demonstrate that we can precisely measure the resistance of individual ca. $20 \mathrm{~nm}$ wide fins and that we can correlate the measured variations in fin resistance with variations in their nanometric width. Due to the demonstrated high precision of the technique, this opens the prospect for the use of $\mu 4 \mathrm{PP}$ in electrical critical dimension metrology.
\end{abstract}

\section{Introduction}

The transition from planar to three-dimensional transistor architectures such as the fin field-effect transistor (finFET) [1] has raised the need for measuring the electrical properties of nanometer-wide conducting features [2]. Recently, it has been shown that the micro four-point probe ( $\mu 4 \mathrm{pp}$ ) technique, which is commonly used for sheet resistance measurements on blanket mate- rials or relatively large pads (larger than $80 \times 80 \mu \mathrm{m}^{2}$ ) [3-5], provides a solution to this requirement [6]. The $\mu 4 \mathrm{pp}$ technique was demonstrated to provide (sheet) resistance measurements in single fins without the need for dedicated Kelvin resistor or transmission line structures [7]. However, the results demonstrated in [6] focused on isolated fins whereby the fin pitch was 
larger than the contact size of the $\mu 4 \mathrm{pp}$ electrodes such that only one single fin was contacted at a time. Intuitively, this suggests that the technique developed therein fails when trying to measure dense structures where a fin pitch smaller than the apparent contact size of the electrodes is used (see below in Figure 2). In that case, the $\mu 4 \mathrm{pp}$ technique appears to be of limited value in routine semiconductor manufacturing where state-of-the-art chips use much smaller fin pitches [8].

In this paper, we describe further developments of the $\mu 4 \mathrm{pp}$ technique, as implemented by the CAPRES A300 tool, which enable the electrical characterization of single nanometer-wide fins in dense fin arrays ( pitch $<200 \mathrm{~nm}$ ) with high precision and repeatability. First, we describe the general concept of how to establish and control the electrical contact between the metallic (Ni-coated) $\mu 4$ pp electrodes and the semiconducting ( $\mathrm{Si}$ ) fins. Next, we show that, by carefully controlling this process, the electrical contact can be confined to one single fin such that the resistance of individual fins in dense arrays can be measured with a high precision. Finally, we use the technique to determine the electrical resistance of individual fins in a dense array and we demonstrate that the measured resistance correlates with the geometrical width of the fins, as measured with transmission electron microscopy (TEM). Due to the demonstrated high precision, a critical dimensional sensitivity of ca. $0.5 \mathrm{~nm}$ could be achieved.

\section{Experimental}

Before discussing the electrical contact between the $\mu 4 \mathrm{pp}$ electrodes and an individual fin, a general description of a $\mu 4 p$ p measurement on large blanket semiconducting samples is needed. The $\mu 4 p p$ electrodes comprise four Ni-coated Si cantilevers with a spacing of $8 \mu \mathrm{m}$ and a contact size $d_{\text {contact }} \approx 300 \mathrm{~nm}[6,9,10]$. In a $\mu 4 \mathrm{pp}$ measurement, the electrodes are landed on the sample surface after which a current $I_{\text {in }}$ is injected into the investigated sample via two of the electrodes while the induced voltage drop $V$ is measured between the other two electrodes. Initially, however, the native oxides present both on the semiconducting material and the $\mathrm{Ni}$-coated electrodes act as highly resistive barriers and therefore prevent any electrical contact [11]. To establish the electrical contact, the $\mu 4 \mathrm{pp}$ technique uses the so-called punchthrough current, i.e., a short current pulse of magnitude $I_{\text {pulse }}$ applied between two electrodes, which causes the breakdown of the native oxide barrier [12-14] and hence creates the conductive path required to inject $I_{\text {in }}$ into the investigated material. Empirically, it is observed that the magnitude of $I_{\text {pulse }}$ must be chosen larger than a certain threshold current ( $I_{\text {threshold }}$, typically $>100 \mu \mathrm{A}$ for blanket materials) in order to reduce the contact resistance $R_{\mathrm{C}}$ between the electrodes and the sample and hence activate the required electrical contact.
The given description of the punch-through mechanism is also valid for more confined structures, such as fins. This does, however, require some additional considerations, starting with the distinction between isolated and dense fins. First, for isolated fins (Figure 1a), i.e., fins are separated by a distance (= pitch) larger than $d_{\text {contact }}$, the procedure is identical to the previously described case of blanket materials. The electrical contact is indeed created, i.e., contacts $j=1,2,3,4$ are activated, when $I_{\text {pulse }} \geq I_{\text {threshold }}$ and the electrical resistance $R_{\text {fin }}$ of the region of the fin included between the two inner contacts is readily obtained from the ratio $R_{\text {fin }}=V / I_{\text {in }}$ [6]. Secondly, in the more complex case of dense fins, i.e., fin pitch $<d_{\text {contact }}$, the $\mu 4 \mathrm{pp}$ electrodes can physically contact multiple fins at the same time. For simplicity, this paper only considers the case of two fins physically contacted by the electrodes (Figure 1b). In this situation, electrical contact is formed on both fins, i.e., contacts $j=1$, $2, \ldots, 8$ are activated, when the magnitude of $I_{\text {pulse }}$ is similar as used on blanket materials. The measured resistance is then determined by the ratio between the two currents $I_{\text {in } 1}$ and $I_{\text {in2 }}$ injected into the two electrically connected fins. Since this ratio depends on the contact resistances $R_{\mathrm{C} j}(j=1,4,5,8)$, this leads to a high measurement variability, i.e., a loss in precision [3]. As a consequence, in order to precisely determine $R_{\text {fin }}$ in a dense fin array, $I_{\text {pulse }}$ should be carefully controlled ( $\left.I_{\text {pulse }}<2 \times I_{\text {threshold }}\right)$ to only allow for the formation of electrical contact to one single fin, i.e., only contacts $j=1,2,3,4$ or $j=5,6,7,8$ are activated. On top of that, to make sure that all four electrodes indeed form electrical contact with the same fin, the punch-through mechanism between electrode pairs must be

\section{(a) Isolated fins (b) Dense fins}
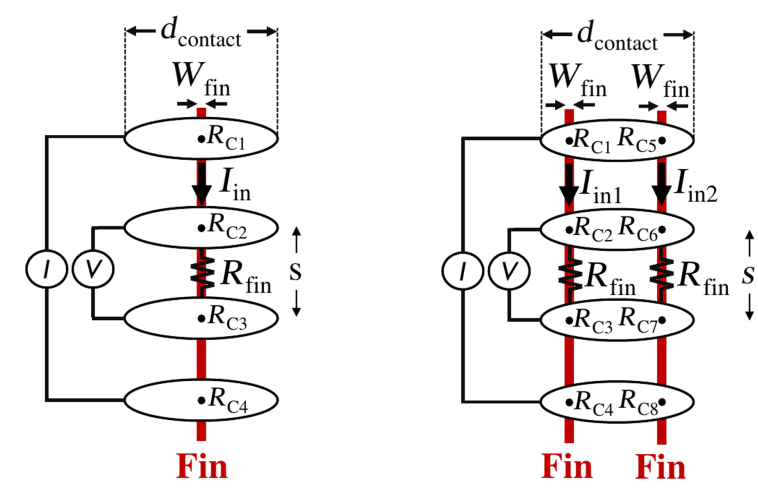

Figure 1: Top-view schematic of the four $\mu 4 p p$ electrodes landed on (a) a single fin and (b) two fins. The electrode contact size and the contact resistance for each electrode-fin contact are, respectively, indicated by $d_{\text {contact }}$ and $R_{\mathrm{Cj}}(j=1,2, \ldots, 8)$. Note that all contact resistances are initially considered to be highly resistive because the native oxides present on both the fins and electrodes prevent current flow into the fin. $R_{\text {fin }}$ is defined as the resistance of the fin between the two inner contacts, i.e., $R_{\text {fin }}=R_{s} \times s / W_{\text {fin, }}$ where $s$ is the distance between the two inner contacts and $W_{\text {fin }}$ is the fin width. 
sequenced properly. For example, when first applying the punch-through mechanism on the top two electrodes, which then form electrical contact with the left fin in Figure 1b, i.e., contacts $j=1$ and $j=2$ are activated, the next electrode pair should use an already activated contact to make sure the contacts on the same fin, i.e., $j=3$ or $j=4$, are activated next. Note that, however, the exact behavior of the electrical contact formation on dense fins is still not fully understood and a more thorough description would also include pulse duration, peak voltage, and material properties.

\section{Results and Discussion}

The experimental demonstration of using the punch-through current $I_{\text {pulse }}$ to individually contact single Si fins in dense arrays is shown in Figure 2a, where the measured $R_{\text {fin }}$ is plotted as a function of the fin width $W_{\text {fin }}$ after using a high $(100 \mu \mathrm{A})$ or low $(25 \mu \mathrm{A})$ punch-through current to form the electrical contact. To highlight the impact of the fin pitch, we have additionally separated the isolated and dense fins, assuming the approximately $300 \mathrm{~nm}$ physical contact size of the electrodes as measured with scanning electron microscopy (SEM) [9]. It can be observed that, while $I_{\text {pulse }}$ does not affect the precision on isolated fins (red and blue triangles), for dense fins a major improvement in precision can be achieved by decreasing the punch-through current from $100 \mu \mathrm{A}$ (red diamonds) to $25 \mu \mathrm{A}$ (blue diamonds). Based on the previous theoretical considerations, the improvement in precision is achieved by restricting the electrical contact to one single fin despite the electrode being in physical contact with two fins. To show this improvement more clearly, the relative standard deviation of the measured values of $R_{\text {fin }}$ can be plotted against the fin pitch, as shown in Figure $2 \mathrm{~b}$. Excitingly, the precision of the $25 \mu \mathrm{A}$ punch-through current measurement remains stable at around $3 \%$, making the measurement feasible even for fin pitch much smaller than $d_{\text {contact }}$. Note that Figure 2 also shows that the fin width has no impact on the measurement precision.

The ability to probe individual fins in dense arrays allows us to exploit the high precision of the $\mu 4 p p$ tool [15] to electrically characterize nanometer-wide fins regardless of the fin pitch. To demonstrate this, Figure 3 shows that we can now measure variations in fin resistance induced by nanometric variations in fin width in a dense array of narrow Si fins. For this, we used an array of ten ca. $20 \mathrm{~nm}$ wide Si fins implanted with B $\left(3 \times 10^{15} \mathrm{~cm}^{-2}, 5 \mathrm{kV}\right)$ and laser-annealed three times at $1150{ }^{\circ} \mathrm{C}$. Note that $W_{\text {fin }}$ is assumed constant, i.e., the very small tapering of the fins along the shallow (ca. $60 \mathrm{~nm}$ ) implant depth [6] is ignored. These fins, having a pitch of $200 \mathrm{~nm}$, were measured individually by using a punch-through current of $25 \mu \mathrm{A}$ to restrict the electrical contact to a single fin. Moreover, by running the $\mu 4 \mathrm{pp}$ measurement over the fin array with a step size of ca. $25 \mathrm{~nm}$, we could assign the measured values of $R_{\mathrm{fin}}$ to each specific fin. As can be observed for the four out of ten fins shown in Figure $3 \mathrm{a}, R_{\mathrm{fin}}$ varies in accordance with the fin width measured by TEM. Note that the error in Figure $3 \mathrm{a}$ is $3.0 \%$ for each fin, which was obtained by taking the lowest precision achieved out of all ten measured fins. Since $R_{\text {fin }}$ is obtained by taking the average of several subsequent measurements, the precision includes the variation in the exact position of the electrical contact points for each landing of the electrodes, i.e., a variation in contact spacing $s$, which may result both from a variation in the electrode positioning itself and from the exact location of the small electrical contact under the wider electrode. Additionally, using the widths measured with TEM (a)

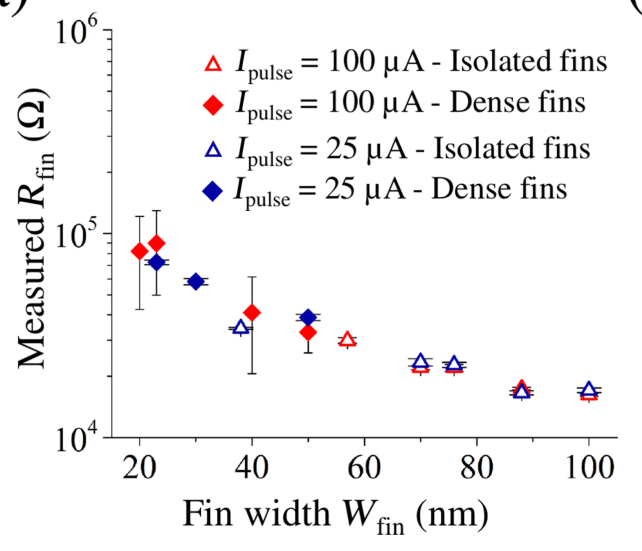

(b)

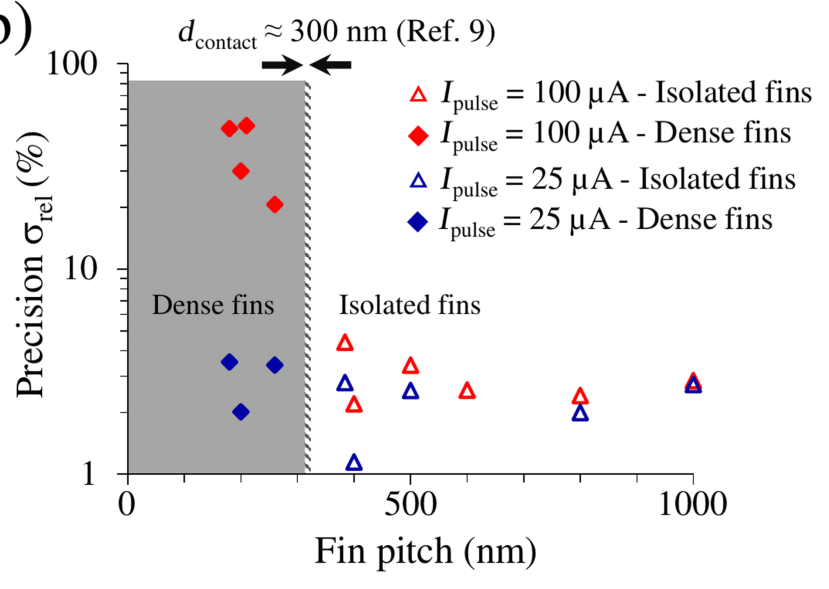

Figure 2: (a) Measured fin resistance $R_{\text {fin }}$ as a function of fin width $W_{\text {fin }}$ on isolated (triangle) and dense (diamond) fins using high (red) and low (blue) punch-through currents. (b) Relative standard deviation of the measured values of $R_{\text {fin }}$ of Figure $2 \mathrm{a}$ as a function of the fin pitch. When using a low punch-through current (blue), the relative standard deviation remains stable $(\leq 3 \%)$ regardless of fin pitch, indicating that the electrical contact remains restricted to a single fin, even in the grey area where the electrodes are in physical contact with more than one fin. 
(a)

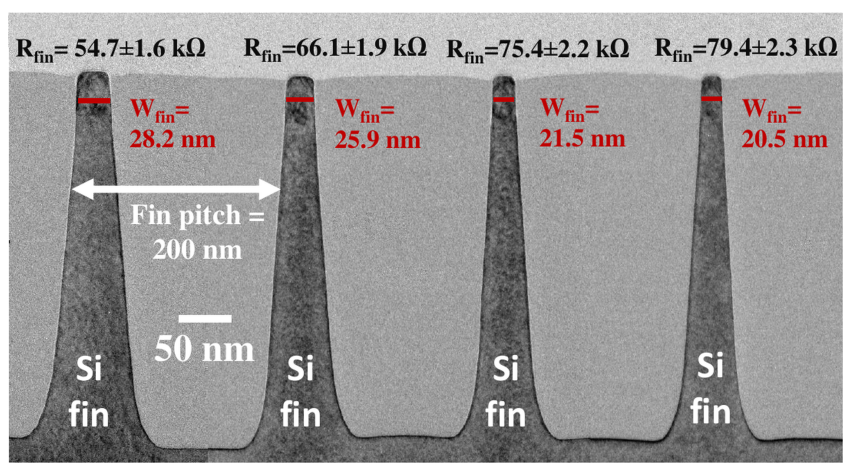

(b)

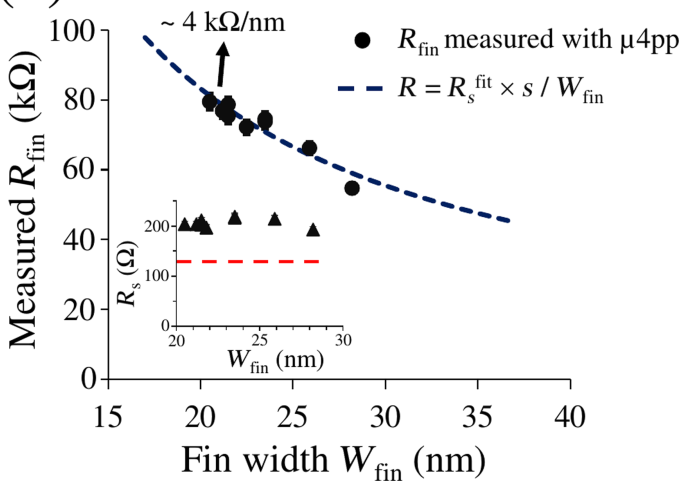

Figure 3: (a) TEM image of four ca. $20 \mathrm{~nm}$ wide $\mathrm{Si}$ fins where the measured $R_{\mathrm{fin}}$ is indicated on top of each fin. The measured values of $R_{\mathrm{fin}}$ correlate to the respective fin width $W_{\text {fin }}$ according to $R_{\text {fin }}=R_{S}^{\text {fin }} \times s / W_{\text {fin }}$. The error for each fin refers to the lowest precision (3.0\%) achieved on all measured fins. (b) Measured fin resistance as a function of $W_{\text {fin }}$ fitted to a constant sheet resistance $R_{s}^{\text {fin }}$ using the relation $R_{\text {fin }}=R_{s}$ fin $\times s / W_{\text {fin }}($ using $s=8 \mu \mathrm{m}$ ). The slope of the fitted curve at $W_{\text {fin }}=20 \mathrm{~nm}$ is indicated (ca. $4.0 \mathrm{k} \Omega / \mathrm{nm}$ ). (inset) Sheet resistance $\left(R_{S}{ }^{\text {fin }}\right)$ of the ten Si fins obtained using the inversed relation $R_{s}^{\text {fin }}=R_{\text {fin }} \times W_{\text {fin }} / s$, plotted against fin width $W_{\text {fin }}$. For comparison, the dashed red line shows the low sheet resistance $R_{s}$ pad $=135 \Omega$ measured on a large pad of the same material as the fins.

and the relation $R_{\text {fin }}=R_{S}^{\text {fin }} \times s / W_{\text {fin }}$ (using $s=8 \mu \mathrm{m}$ ), the inset of Figure $3 \mathrm{~b}$ shows that all ten fins have the same sheet resistance $R_{S}{ }^{\text {fin }} \approx 200 \Omega /$ sq, indicating that the observed variations in $R_{\text {fin }}$ are indeed caused by variations in fin width. This allows us to evaluate the sensitivity of the technique by plotting the measured values of $R_{\text {fin }}$ as a function of the fin width (Figure $3 \mathrm{~b}$ ) and subsequently fitting the data with a constant sheet resistance $R_{S}{ }^{\text {fin }}$ using the relation $R_{\text {fin }}=R_{S}{ }^{\text {fin }} \times s / W_{\text {fin }}$. By comparing the slope of the fitted curve at $W_{\text {fin }}=20 \mathrm{~nm}$ (ca. $4 \mathrm{k} \Omega / \mathrm{nm}$ ) to the achieved precision (ca. $2.3 \mathrm{k} \Omega$, Figure $3 \mathrm{a}$ ), we can deduce that the technique has a sensitivity to variations in fin width down to about $0.5 \mathrm{~nm}$, opening the prospects for its use in electrical critical dimension metrology. As also interestingly shown in the inset of Figure $3 \mathrm{~b}$, the measured $R_{S}$ fin is higher than the sheet resistance $R_{S}{ }^{\text {ad }}=135 \Omega$ (dashed red line) measured in a large $80 \times 80 \mu \mathrm{m}^{2}$ pad having undergone the same implantation and annealing treatment. This increase in sheet resistance when going to nanoscale elongated geometries was expected and understood to originate from the presence of interface states as well as defects at the fin sidewalls [6].

\section{Conclusion}

This paper demonstrates the capability of $\mu 4 p p$ to electrically characterize individual nanometer-wide Si fins in dense arrays regardless of fin pitch. By carefully controlling the electrical contact, we were able to measure the resistance of individual ca. $20 \mathrm{~nm}$ wide fins in dense arrays even though the $\mu 4 \mathrm{pp}$ electrodes physically contact more than one fin. Thanks to the high precision of the measurements, the correlation between measured resistance and nanometer-scale variations in fin width could be demonstrated with a sensitivity as small as $0.5 \mathrm{~nm}$.

\section{Acknowledgements}

This project has received funding from the "European Union's Horizon 2020 research and innovation program" under grant agreements No 688225 and No 692527.

\section{ORCID ${ }^{\circledR}$ iDs}

Steven Folkersma - https://orcid.org/0000-0002-0166-6037 Dirch H. Petersen - https://orcid.org/0000-0002-9309-4186 Ole Hansen - https://orcid.org/0000-0002-6090-8323

\section{References}

1. Hisamoto, D.; Lee, W.-C.; Kedzierski, J.; Takeuchi, H.; Asano, K.; Kuo, C.; Anderson, E.; King, T.-J.; Bokor, J.; Hu, C. IEEE Trans. Electron Devices 2000, 47, 2320-2325. doi:10.1109/16.887014

2. Schroder, D. K. Series Resistance, Channel Length and Width, and Threshold Voltage. Semiconductor Material and Device Characterization, 3rd ed.; Wiley-Interscience: New York, NY, U.S.A., 2006; pp 185-250.

3. Petersen, D. H.; Hansen, O.; Hansen, T. M.; Bøggild, P.; Lin, R.; Kjær, D.; Nielsen, P. F.; Clarysse, T.; Vandervorst, W.; Rosseel, E.; Bennett, N. S.; Cowern, N. E. B. J. Vac. Sci. Technol., B 2010, 28, C1C27. doi:10.1116/1.3224898

4. Clarysse, T.; Bogdanowicz, J.; Goossens, J.; Moussa, A.; Rosseel, E.; Vandervorst, W.; Petersen, D. H.; Lind, R.; Nielsen, P. F.; Hansen, O.; Merklin, G.; Bennett, N. S.; Cowern, N. E. B. Mater. Sci. Eng., B 2008, 154-155, 24-30. doi:10.1016/j.mseb.2008.09.038

5. Thorsteinsson, S.; Wang, F.; Petersen, D. H.; Hansen, T. M.; Kjær, D. Lin, R.; Kim, J.-Y.; Nielsen, P. F.; Hansen, O. Rev. Sci. Instrum. 2009, 80, 053902. doi:10.1063/1.3125050

6. Bogdanowicz, J.; Folkersma, S.; Sergeant, S.; Schulze, A.; Moussa, A.; Petersen, D. H.; Hansen, O.; Henrichsen, H. H.; Nielsen, P. F.; Vandervorst, W. Phys. Status Solidi A 2018, 215, 1700857. doi:10.1002/pssa.201700857 
7. Schroder, D. K. Resistivity. Semiconductor material and device characterization, 3rd ed.; Wiley-Interscience: New York, NY, U.S.A., 2006; pp 1-59.

8. Natarajan, S.; Agostinelli, M.; Akbar, S.; Bowonder, A.; Chikarmane, V.; Chouksey, S.; Dasgupta, A.; Fu, Q.; Ghani, T.; Giles, M.; Govindaraju, S.; Grover, R.; Han, W.; Hanken, D.; Haralson, E.; Haran, M.; Heckscher, M.; Heussner, R.; Jain, P.; James, R.; Jhaveri, R.; Jin, I.; Kam, H.; Karl, E.; Kenyon, C.; Liu, M.; Luo, Y.; Mehandru, R.; Morarka, S.; Neiberg, L.; Packan, P.; Paliwal, A.; Parker, C.; Patel, P.; Patel, R.; Pelto, C.; Pipes, L.; Plekhanov, P.; Prince, M.; Rajamani, S.; Sandford, J.; Sell, B.; Sivakumar, S.; Smith, P.; Song, B.; Tone, K.; Troeger, T.; Wiedemer, J.; Yang, M.; Zhang, K. A 14nm logic technology featuring 2nd-generation FinFET, air-gapped interconnects, self-aligned double patterning and a 0.0588 $\mu \mathrm{m} 2$ SRAM cell size. In 2014 IEEE International Electron Devices Meeting (IEDM), San Francisco, CA, U.S.A., Dec 15-17, 2014; pp 71-73. doi:10.1109/IEDM.2014.7046976

9. Petersen, D. H.; Hansen, O.; Hansen, T. M.; Petersen, P. R. E.; Bøggild, P. Microelectron. Eng. 2008, 85, 1092-1095. doi:10.1016/j.mee.2007.12.077

10. Wang, F.; Petersen, D. H.; Jensen, H. V.; Hansen, C.; Mortensen, D.; Friis, L.; Hansen, O. J. Micromech. Microeng. 2011, 21, 085003. doi:10.1088/0960-1317/21/8/085003

11. Morita, M.; Ohmi, T.; Hasegawa, E.; Teramoto, A. Jpn. J. Appl. Phys. 1990, 29, L2392-L2394. doi:10.1143/JJAP.29.L2392

12. Lombardo, S.; Stathis, J. H.; Linder, B. P.; Pey, K. L.; Palumbo, F.; Tung, C. H. J. Appl. Phys. 2015, 98, 121301. doi:10.1063/1.2147714

13. Verweij, J. F.; Klootwijk, J. H. Microelectron. J. 1996, 27, 611-622. doi:10.1016/0026-2692(95)00104-2

14. Klein, N.; Gafni, H. IEEE Trans. Electron Devices 1966, 13, 281-289. doi:10.1109/T-ED.1966.15681

15. Wang, F.; Petersen, D. H.; Hansen, T. M.; Henriksen, T. K.; Bøggild, P.; Hansen, O. J. Vac. Sci. Technol., B 2010, 28, C1C34. doi:10.1116/1.3224889

\section{License and Terms}

This is an Open Access article under the terms of the Creative Commons Attribution License (http://creativecommons.org/licenses/by/4.0), which permits unrestricted use, distribution, and reproduction in any medium, provided the original work is properly cited.

The license is subject to the Beilstein Journal of Nanotechnology terms and conditions: (https://www.beilstein-journals.org/bjnano)

The definitive version of this article is the electronic one which can be found at: $\underline{\text { doi:10.3762/bjnano.9.178 }}$ 